\title{
Torsión segmentaria primaria del epiplón mayor como causa poco frecuente de abdomen agudo quirúrgico.
}

Primary segmental torsion of the greater omentum as a rare cause of acute surgical abdomen.

Dr. Julio Efrain Naula Guamán. ${ }^{1}$, Dr. Jhon Paúl Ortiz Torres. ${ }^{2}$ \& Dra. Fanny Karina León Loaiza. $^{3}$

Abstract.

DOI: https://doi.org/10.33262/cienciadigital.v2i3.172

The torsion or segmental infarction of the omentum is a rare cause of acute abdomen with nonspecific symptoms that can be confused with other intra-abdominal surgical pathologies such as appendicitis, diverticulitis, cholecystitis among others, its etiology is unknown but different risk factors have been associated with it. classified as predisposing or triggering, primary or secondary and external or internal; The diagnosis is usually made by an exploratory laparotomy. The following is the clinical case of a patient with torsion of omentum managed in the Basic Hospital of Yantzaza, it is a 24-year-old male patient who came to the emergency room due to presenting, without any apparent cause, moderate diffuse abdominal pain. intensity of more than 48 hours of evolution the pain at 36 hours intensifies and localizes in the flank and right iliac fossa was evaluated clinically diagnosing an acute surgical abdomen because of acute appendicitis so it was treated by exploratory laparotomy finding a segment torsion right of the greater omentum.

Keywords: Omental Torsion, Acute Abdomen, Exploratory Laparotomy

\section{Resumen.}

La torsión o infarto segmentario del epiplón es una causa poco frecuente de abdomen agudo con síntomas inespecíficos que pueden confundirse con otras

\footnotetext{
${ }^{1}$ Hospital Básico Yantzaza, Zamora Chinchipe, Ecuador, jnaulagdr@ hotmail.com

${ }^{2}$ Hospital Básico Yantzaza, Zamora Chinchipe, Ecuador, jortiz_311@ @otmail.com

${ }^{3}$ Universidad Nacional de Loja, Loja, Ecuador, karyn_leon1@ @otmail.com
} 
patologías quirúrgicas intraabdominales como apendicitis, diverticulitis, colecistitis entre otras, su etiología es desconocida pero se han asociado diferentes factores de riesgo que se han clasificado como predisponentes o desencadenantes, primarios o secundarios y externos o internos; el diagnóstico generalmente se realiza mediante una laparotomía exploratoria. A continuación se presenta el caso clínico de una paciente con torsión de epiplón manejado en el Hospital Básico de Yantzaza, se trata de un paciente de 24 años de edad de sexo masculino que acudió a emergencias por presentar sin ninguna causa aparente dolor abdominal difuso de moderada intensidad de más de 48 horas de evolución el dolor a las 36 horas se intensifica y localiza en flanco y fosa iliaca derecha fue evaluado clínicamente diagnosticando un abdomen agudo quirúrgico a causa de apendicitis aguda por lo que fue tratado por laparotomía exploratoria encontrándose una torsión del segmento derecho del epiplón mayor.

Palabras Claves: Torsión Omental, Abdomen Agudo, Laparotomía Exploratoria.

\section{Introducción.}

La torsión o infarto del epiplón es una patología poco frecuente esta entidad fue descrita por primera vez por Eitel ${ }^{1}$ en 1899 pero hasta 1981 sólo 233 casos habían sido reportados en la literatura médica. La probabilidad de un caso de torsión de epiplón contra un caso de apendicitis aguda es de 4:1000 y la incidencia reportada varía de $0.0016 \%$ hasta $0.37 \%{ }^{4,5}$. Generalmente se presenta en la cuarta o quinta década de vida. La relación entre hombres y mujeres es de $5: 1$, y solo un $0.1 \%$ de los casos reportados son en niños. Se considera que la edad pediátrica en la que se puede presentar es de 9 a 16 años, excluyendo a niños menores de 4 años, debido a su poco tejido adiposo ${ }^{6,7,8}$.

La etiología de la torsión de epiplón es desconocida por lo que existen diversas hipótesis, Donhauser y Loke clasificaron la torsión de epiplón en primaria y secundaria. La primaria se debe a un pedículo del epiplón muy angosto o muy largo y la lesión vascular debido a exceso de grasa; la secundaria ocasionada por trauma externo, ejercicio violento o movimiento peristáltico muy acelerado, quistes, tumores en el epiplón, hernias, adherencias. ${ }^{9,10,11}$ Adams $^{12}$ asoció a la torsión omental con factores desencadenantes y factores predisponentes de los cuales sobresalen las variaciones anatómicas, obesidad y distribución de la circulación del epiplón ${ }^{13}$.

La torsión de epiplón se desarrolla por un conjunto de giros alrededor de su propio eje comprimiendo la irrigación arterial epiploica distal, lo cual causa dolor abdominal del lado comprometido5. La torsión omental puede darse tanto en el cuadrante inferior izquierdo como en el derecho, predominando en este último por ser de mayor longitud hasta en $90 \%$ de los casos, siendo más fácil que rote hacia ese lado. 
Debido a que el dolor abdominal es más frecuente del lado derecho, puede confundirse con otras entidades como colecistitis aguda, apendicitis aguda, úlcera duodenal perforada o pseudomixoma peritoneal. Durante el proceso de torsión de epiplón se puede provocar obstrucción venosa, edema y compromiso arterial, lo que lleva a infarto omental ${ }^{4,8,11,12}$.

El diagnostico preoperatorio de esta patología es difícil por lo inespecífico de la sintomatología ante ello se torna importante analizar los diferentes casos reportados y así determinar un patrón de presentación de la enfermedad y así poder reconocer la opción de tratamiento adecuada que puede ser conservadora o quirúrgica. Estudios realizados de reportes de casos de torsión de epiplón describen la difusión del uso en urgencias de la ecografía y tomografía computarizada para conseguir el diagnóstico preoperatorio y así garantizar la idoneidad de la abstención quirúrgica en casos seleccionados.

Debido a que en casi todos los casos la patología se presenta con un cuadro similar a la apendicitis aguda otros autores han investigado los síntomas relacionados con apendicitis aguda cuyos resultados fueron que la sintomatología fue similar a la de la apendicitis aguda con ciertas peculiaridades como un más largo período de evolución en el momento del diagnóstico, una fiebre y leucocitosis menores que en el caso de una apendicitis con esas horas de evolución y, en algunos casos, la ausencia de vómitos determinándose que en todos los casos de torsión primaria el diagnóstico clínico fue de apendicitis aguda.

\section{Descripción del caso clínico:}

Se trata de una paciente de 24 años de edad quien consulta al servicio de urgencias del Hospital Básico de Yantzaza de la provincia de Zamora Chinchipe por presentar dolor abdominal difuso de moderada intensidad de más de 48 horas de evolución que luego a las 36 horas el dolor se intensifica y localiza en flanco y fosa iliaca derecha el paciente no tiene antecedentes patológicos de importancia en el examen físico: Peso $81.6 \mathrm{Kg}$; IMC 27,8; TA: 130/84; FC 98: FR 26; T 37. Dolor localizado en flanco y fosa iliaca derechos, con contractura muscular y reacción peritoneal. Laboratorio: Hemoglobina 14,2; Hematocrito 43,5; Leucocitos 6580; Neutrófilos 60\%; Urea 27,3; Creatinina 1,11; TGP 10,9: TGO 72,8; Fosfatasa Alcalina 91; Amilasa 68; PCR 12. Se diagnosticó un abdomen agudo inflamatorio y decidió realizar una laparotomía exploradora con sospecha diagnóstica de apendicitis aguda.

En la cirugía los hallazgos fueron: 
Figura a. masa friable adosada a la pared anterior del abdomen, sobre el colon ascendente, de 7 x $7 \mathrm{~cm}$, con escaso liquido inflamatoria peri lesional.

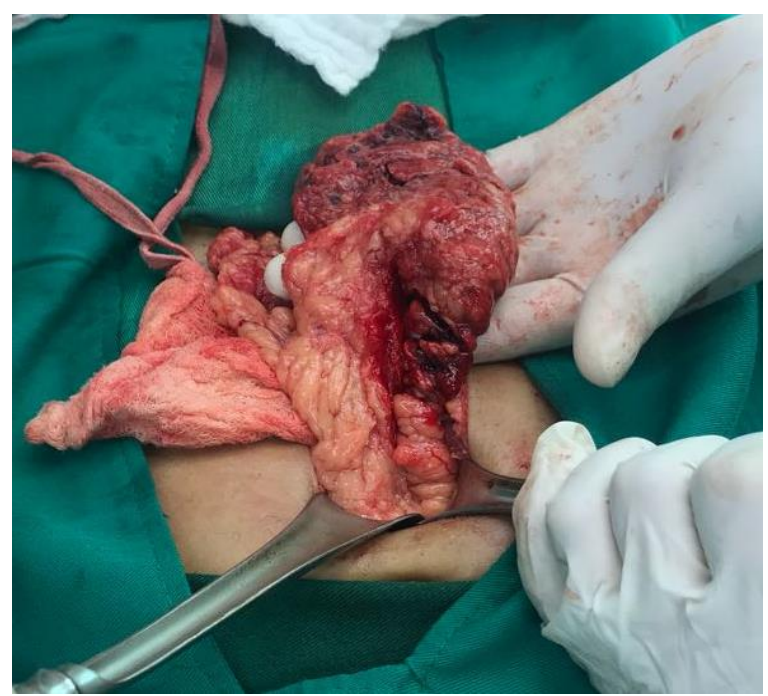

Autor: Grupo de investigación.

Figura 2. Identificamos que dicha lesión proviene del segmento derecho del epiplón mayor, mismo que ha rotado sobre su propio eje varias veces.

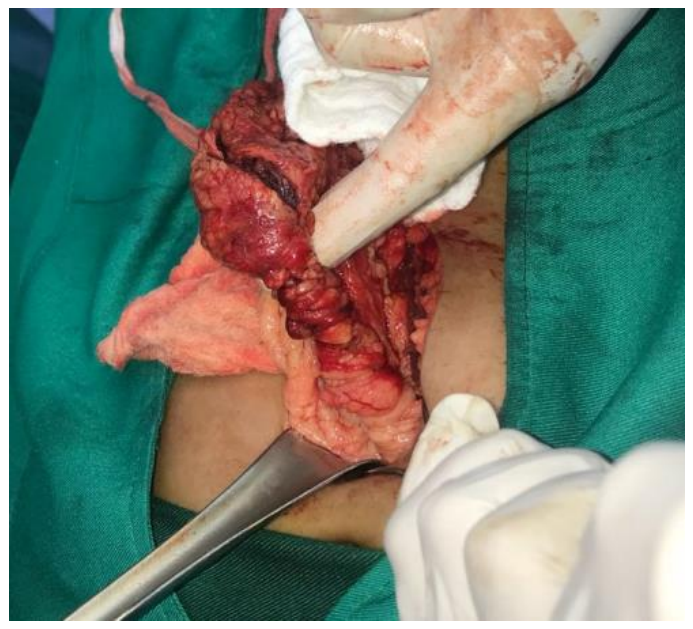

Autor: Grupo de investigación. 
Figura c. realizamos omentectomía parcial y apendicectomía complementaria.

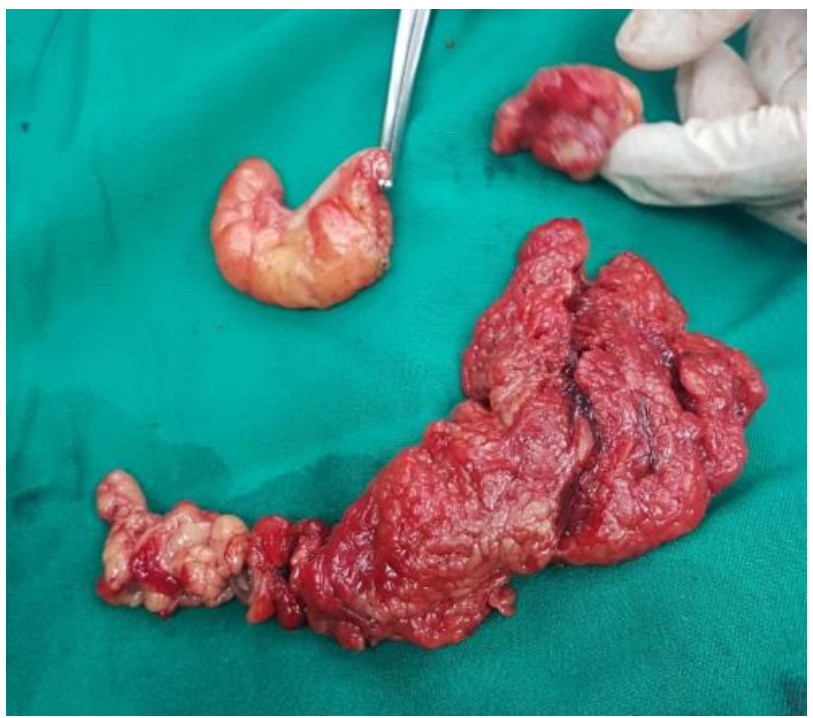

Autor: Grupo de investigación.

Figura d. exploramos la pieza extraída y observamos signos de necrosis por infarto distal a la torsión omental.

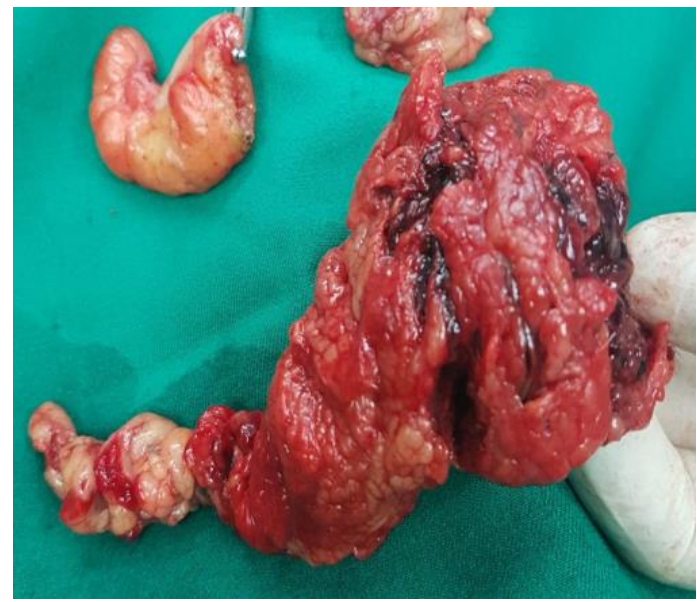

Autor: Grupo de investigación

El paciente presenta una evolución satisfactoria, recibe profilaxis antibiótica, se da el alta a las 48 horas del post operatorio, el informe de anatomía patológica no informa sobre alguna causa secundaria de la torsión del epiplón.

\section{Discusión.}

Dentro de la medicina y sobre todo, dentro de amplio campo de la cirugía, el diagnóstico de una afección que requiera un tratamiento quirúrgico se convierte en un verdadero reto, 
decidiendo intervenir a un paciente bajo una sospecha diagnóstica frecuente como es una apendicitis aguda, sin embargo nos encontramos con otra entidad poco común como es la torsión parcial del epiplón mayor; que revisando la literatura internacional en la actualidad no son más de 300 casos reportados; incluyendo un caso publicado hace 1 año en el hospital del Cantón Zamora de la provincia de Zamora Chinchipe.

La etiología de la torsión primaria de epiplón se desconoce; se han descrito varios factores relacionados con la predisposición de la enfermedad. Algunos autores como Donhauser y Loke $^{11}$, Adams ${ }^{12}$, Mallick y Al-Bassam ${ }^{14}$ han propuesto clasificaciones donde sugiere al sobrepeso como factor predisponente. Estudios a 30, 20 y 10 años de análisis en Toronto, Canadá y Melbourne, Australia, muestran un importante incremento en la incidencia de la torsión epiploica de 0.215 y $0.166 \%$ respectivamente, probablemente asociado al aumento de obesidad ${ }^{15}$. En el caso del paciente reportado en este hospital se determinó que su IMC era de 27.8 lo cual indica sobrepeso que podría ser un factor predisponente para el desarrollo de esta patología.

El diagnóstico preoperatorio es complejo por la ambigüedad de los síntomas con los que se presenta, entre ellos se encuentra dolor abdominal, dolor en fosa ilíaca derecha, hipersensibilidad en la zona afectada, leucocitosis y fiebre menor de 38 。 $\mathrm{C}^{16-17}$. Más del 50\% presenta abdomen distendido, hipersensibilidad al tacto, náuseas, vómitos, leucocitosis y alza térmica que no suele alcanzar los $38 \circ \mathrm{C}$, a diferencia de la apendicitis aguda ${ }^{18}$. Los estudios de imagen no muestran ningún dato radiológico que indique torsión de epiplón, confundiéndose con lipoma, liposarcoma, apendicitis, vólvulo intestinal, necrosis de tejido adiposo y paniculitis; O־guzkurt et al. ${ }^{19}$ reconoció como hallazgo universal con el ultrasonido la presencia de líquido estéril y serosanguinolento dentro de la cavidad peritoneal.

Actualmente el ultrasonido muestra una cantidad mínima de líquido intraabdominal; el aspecto ecográfico común es una masa que se adhiere a la pared abdominal anterior de forma ovoide, la cual se describe como una lesión hiperecoica con bordes hipoecoicos, incompresible y se relaciona a hipersensibilidad ubicada en la lesión 19, 20 .

En la resonancia magnética se pueden observar estructuras hipointensas lineales, que corresponden al poco flujo de los vasos mesentéricos, dentro de una masa de grasa hiperintensa en T1, se presenta congestión y edema en el epiplón mayor en ponderado $\mathrm{T} 2^{5}$.

Los resultados de la tomografía axial computada (TAC) muestran una masa ovoide, adherida a la pared abdominal, en la región umbilical o anterolateral a la mitad del colon, si la región es medial con respecto al colon ascendente favorece a la torsión omental, si se encuentra propiamente en ciego-colon se relaciona con apendicitis ${ }^{21,22}$. La característica típica de la torsión de epiplón en la TAC es su patrón difuso en forma de remolino fibroso y graso, aunque poco específico para saber si forma parte de un órgano abdominal ${ }^{23}$. 
Además se pueden observar dos signos en la TAC sugestivos de esta enfermedad. En el "signo de pedículo vascular" se observa un punto central del potenciador buque de la mesentérica rodeando con múltiples giros a las ramas mesentéricas de menor tamaño. El "signo torbellino" se describe como una masa de grasa nebulosa con líneas concéntricas grasas, torciendo los vasos sanguíneos dentro del epiplón mayor, que giran alrededor de una línea vascular central ${ }^{16}$; este último signo puede afirmar la torsión del epiplón mayor.

En el tratamiento de la torsión de epiplón se puede optar por una conducta conservadora, en caso de ser torsión de epiplón secundaria, se corrige la condición principal y se da un seguimiento radiológico. El tratamiento quirúrgico consta, en la resección de la porción del epiplón afectado ya sea por laparotomía o por mínimo acceso, de esta manera se evita la posible sepsis y se consigue una menor estancia hospitalaria ${ }^{5}$.

\section{Conclusiones.}

- La torsión de epiplón es una causa poco frecuente de abdomen agudo y se puede confundir con facilidad con otras afecciones por su presentación clínica inespecífica, haciendo complejo su diagnóstico.

- El estudio de imagen recomendado es la tomografía computada que muestra el «signo del torbellino».

- El tratamiento de elección es el abordaje quirúrgico y exéresis del segmento afectado, ya sea por laparotomía o por vía laparoscópica, siendo esta última un abordaje menos invasivo y con menor tiempo de recuperación.

\section{Referencias bibliográficas.}

Eitel GG. Rare Omental Torsion. New York Med Rec. 1899;55:715.

Basson SE, Jones PA. Primary torsion of the omentum. Ann R Coll Surg Engl. 1981;63:132-4.

Sweeney MJ, Blestel GA, Ancalmo N. Primary torsion of the greater omentum. A rare cause of abdominal pain in children. JAMA. 1983;249:3073.

Varjavandi V, Lessin M, Kooros K, Fusunyan R, McCauley R, Gilchrist B. Omental infarction: Risk factors in children. J Pediatr Surg. 2003;38:233-5

Zaleta-Cruz J , Rojas-Méndez J, et al. Torsión de epiplón. Reporte de caso. Academia Mexicana de Cirugía A.C. Masson Doyma México S.A. CC BY-NC-ND, 2015(http://creativecommons.org/licenses/by-nc-nd/4.0/).

Sencan A, Arslan O, Yilmaz O, Ayhan S, Mir E. A rare cause of acute abdominal pain: primary torsion of omentum majus. Turk J Gastroenterol. 2002;13:122---4. 
Sarango Peláez W, Serrano Ortega B, Jaramillo Sisalima P, Torsión omental, a propósito de un caso; servicio de Cirugía General del hospital General Julius Doepfner de la ciudad de Zamora. Universidad Nacional de Loja. Julio 2017.

Baeza Herrera C, Vidrio Padrón F, Mejía Camacho D, et al. Torsión primaria del epiplón. Extraña causa de dolor abdominal. Revista Mexicana de Pediatría; 2010; 77(6); 257-259. http://www.medigraphic.com/rmp

Uscátegui H. Torsión primaria de epiplón. Caso clínico. Departamento de Cirugía General Hospital Universitario de Santander, Universidad Industrial de Santander. Santander, Colombia. Revista Chilena de Cirugía. Vol 62 - No 4, Agosto 2010; pág. 408-411.

Andreuccetti J, Ceribelli C, Manto O, Chiaretti M, Negro P, Tuscano D. Primary omental torsion (POT): a review of literature and case report. World J Emerg Surg. 2011;6:6.

Donhauser JL, Locke D. Primary torsion of omentum: report of six cases. AMA Arch Surg. 1954;69:657---62.

Adams JT. Primary torsion of the omentum. Am J Surg. 1973;126:102-5.

Scabini S, Rimini E, Massobrio A, Romairone E, Linari C, Scordamaglia R, et al. Primary omental torsion: A case report. World J Gastrointest Surg. 2011;3:153---5.

Mallick MS, Al-Bassam AA. Primary omental torsion in children. The predisposing factors and role of laparoscopy in diagnosis and treatment. Saudi Med J. 2006;27:194---7.

Chaudhary D, Rajkarnikar R, Joshi MR, Thapa P, Singh DR, Sharma SK. Omental torsion: a case report. Kathmandu Univ Med J (KUMJ). 2005;3:170---2.

Coppin T, Lipsky D. Twisting and infarction of the entire greater omentum managed by laparoscopy: a report of two cases. Acta Chir Belg. 2006;106:215-7.

Sakamoto N, Ohishi T, Kurisu S, Horiguchi H, Arai Y, Sugimura K. Omental torsion. Radiat Med. 2006;24:373-7.

Stella DL, Schelleman TG. Segmental infarction of the omentum secondary to torsion: ultrasound and computed tomography diagnosis. Australas Radiol. 2000;44:212-5.

O guzkurt P, Kotilo glu E, Tanyel FC, Hicsönmez A. Primary omental torsion in a 6year-old girl. J Pediatr Surg. 1995;30:1700-1

Puylaert JB. Right-sided segmental infarction of the omentum: clinical, US, and CT findings. Radiology. 1992;185:169-72.

Rich RH, Filler RM. Segmental infarction of the greater omentum: a cause of acute abdomen in childhood. Can J Surg.1983;26:241-3. 
Poujade O, Ghiles E, Senasli A. Primary torsion of the greater omentum: case reportReview of Literature: Diagnosis cannot always be performed before surgery. Surg Laparosc Endosc Percutan Tech. 2007;17:54-5.

Naffaa LN, Shabb NS, Haddad MC. CT findings of omental torsion and infarction: case report 


\section{Para citar el artículo indexado.}

Naula J., Ortiz J. \& León F. . (2018). Torsión segmentaria primaria del epiplón mayor como causa poco frecuente de abdomen agudo quirúrgico. Revista electrónica Ciencia Digital 2(3), 503-512. Recuperado desde: http://cienciadigital.org/revistacienciadigital2/index.php/CienciaDigital/article/view/172/15 $\underline{2}$

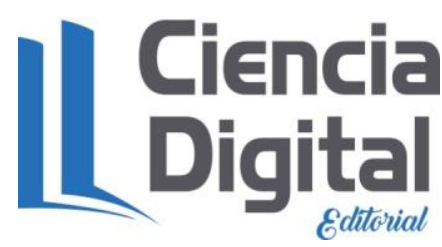

El artículo que se publica es de exclusiva responsabilidad de los autores y no necesariamente reflejan el pensamiento de la Revista Ciencia Digital.

El articulo queda en propiedad de la revista y, por tanto, su publicación parcial y/o total en otro medio tiene que ser autorizado por el director de la Revista Ciencia Digital.
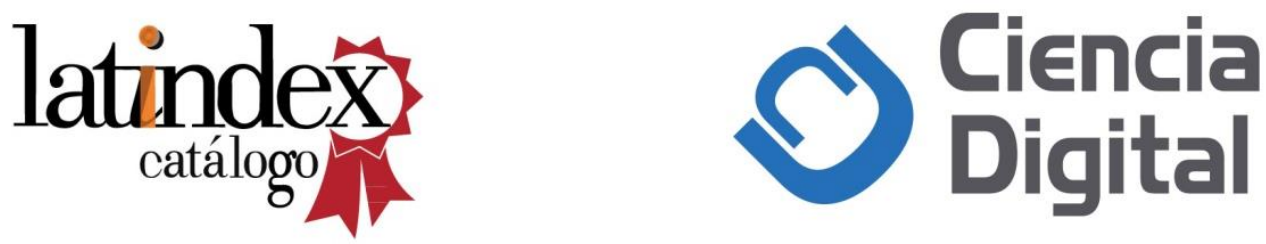